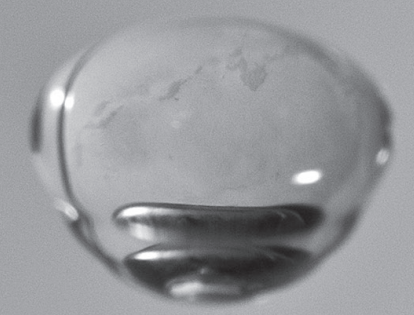

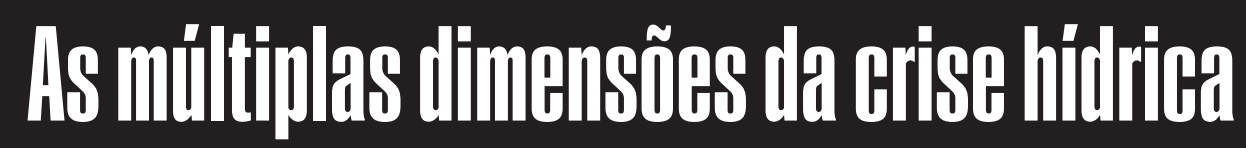

\author{
José Galizia Tundisi
}

Takako Matsumura Tundisi 


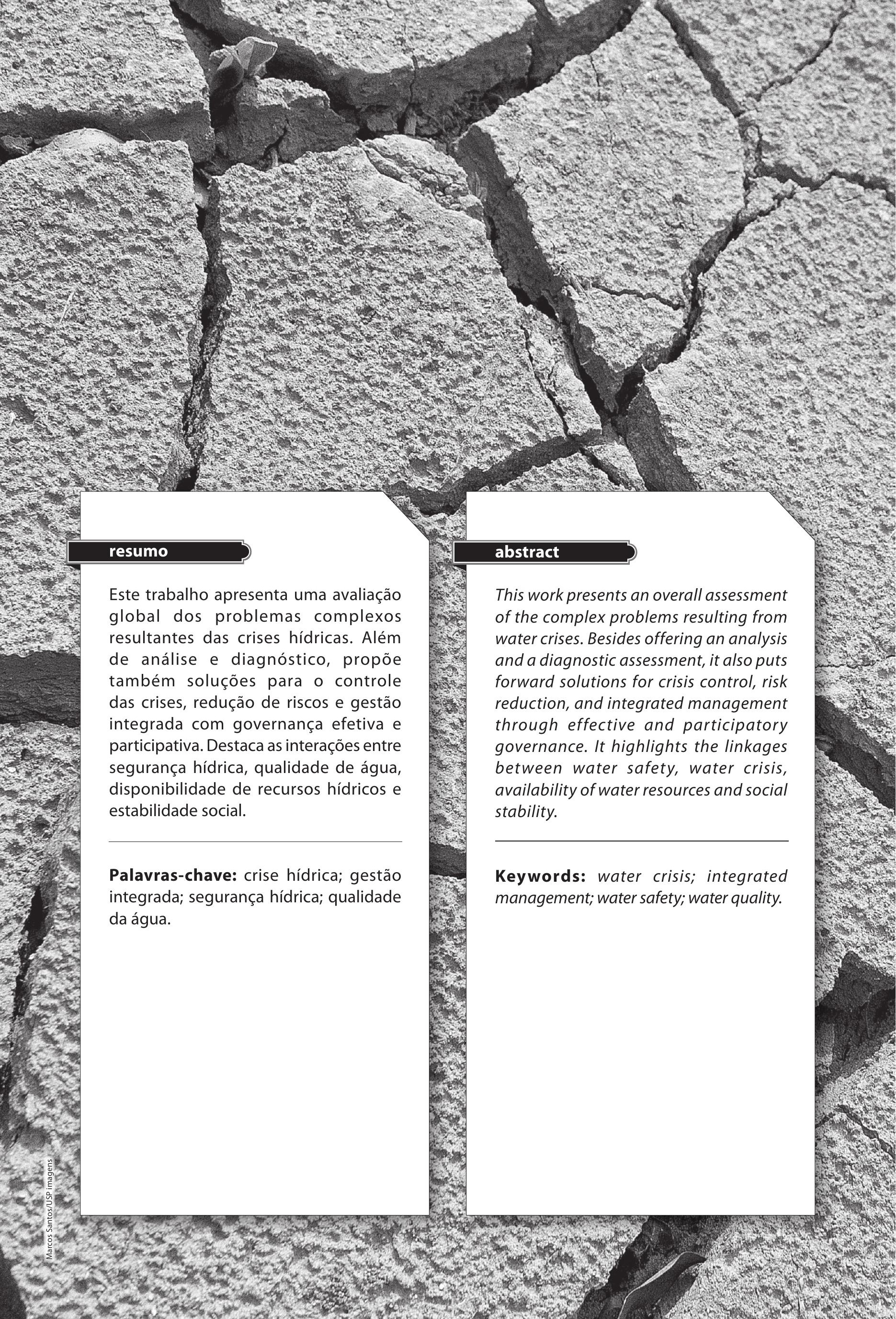




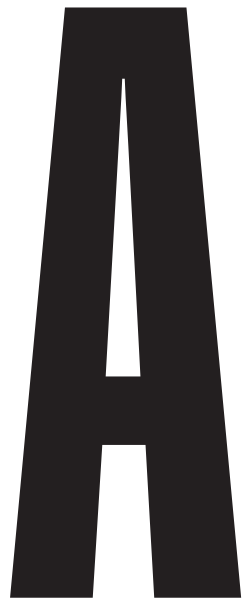

crise hídrica que ocorre em muitas regiões do planeta não é limitada somente a secas prolongadas, mas também a extremos hidrológicos, intensas precipitações, que são igualmente danosas às propriedades, à saúde humana e ao funcionamento dos ecossistemas. Tais crises afetam três componentes essenciais do recurso hídrico, fundamental à qualidade de vida e à sobrevivência da humanidade: a vulnerabilidade a desastres (secas e enchentes); a acessibilidade à água; e a segurança hídrica. As crises hídricas são decorrentes de fenômenos globais (mudanças climáticas e alterações no funcionamento global do clima), ações humanas intensivas, como desmatamento, urbanização, usos do solo, construções de infraestrutura (canais, represas, rodovias), com efeitos sinérgicos que afetam milhões de pessoas. Dentre todas essas ações, a urbanização é um dos principais problemas. Atualmente há 37 megacidades no planeta, cada uma com mais de 10 milhões de habitantes. $\mathrm{O}$ suprimento de água, para essas cidades, é um dos grandes problemas atuais. Além disso, elas produzem milhões de toneladas de detritos, diariamente, os quais, se não tratados, afetam mananciais, águas subterrâneas, rios e lagos. Portanto, as mudanças climáticas, que alteram padrões de precipitação e os ciclos naturais, umidade do solo, balanço hídrico em geleiras, em sinergia com a poluição, desmatamento e usos intensivos do solo, são o grande problema atual e se estenderão por boa parte do século XXI.
As crises hídricas têm múltiplas e complexas dimensões, como se demonstrará neste trabalho.

\section{SEGURANÇA HÍDRICA}

A segurança hídrica é definida, pelo Programa Hidrológico Internacional da Unesco, como a

“[...] capacidade de assegurar a uma população o acesso a quantidades adequadas de água de qualidade aceitável com a finalidade de sustentar a saúde humana e a saúde dos ecossistemas, em uma bacia hidrográfica, e também assegurar proteção eficiente da vida e da propriedade contra desastres relacionados a recursos hídricos - secas, enchentes, deslizamentos, afundamento de solos" (Unesco, IHP, 2012).

Essa definição inclui, portanto, não só a segurança coletiva para as populações humanas, mas a água necessária para o funcionamento dos ecossistemas, para a manutenção dos ciclos e para a sustentabilidade.

JOSÉ GALIZIA TUNDISI é professor titular aposentado da Escola de Engenharia de São Carlos da USP, professor titular da Universidade Feevale (RS) e presidente da Associação Instituto Internacional de Ecologia e Gerenciamento Ambiental.

TAKAKO MATSUMURA TUNDISI é professora titular aposentada da Universidade Federal de São Carlos (UFSCar) e vice-presidente da Associação Instituto Internacional de Ecologia e Gerenciamento Ambiental. 
A segurança hídrica destaca, assim, duas ações fundamentais: água como ameaça à segurança das populações humanas e aos ecossistemas (enchentes, secas, poluição); e água para assegurar suprimento adequado aos múltiplos usos.

\section{AS ATIVIDADES HUMANAS E A CRISE HÍDRICA}

As atividades humanas que afetam os ciclos hidrológicos, a disponibilidade de água e o acesso à água têm se intensificado e se tornado mais complexas desde a metade do século XX. Como os efeitos das mudanças climáticas serão intensificados, os extremos de secas e enchentes aumentarão a vulnerabilidade das populações urbanas e o acesso à água.

Também devem ocorrer alterações da qualidade de água potável, interferindo em usos como recreação, pesca e agricultura, podendo a produção de alimentos ficar comprometida (Watson et al., 1998).

A expansão e a diversificação dos usos múltiplos da água marcaram definitivamente as últimas décadas do século XX. Produção de alimentos, hidroeletricidade, navegação, recreação, agricultura, abastecimento público são usos competitivos da água, para os quais são necessárias governança efetiva, estratégia integrada e definição de prioridades.

As atividades humanas na paisagem, na cobertura vegetal, nos usos do solo, produzindo erosão, sedimentação dos corpos de água, contaminação e poluição em larga escala, estão afetando os recursos hídricos. E as crises hídricas agravam esses efeitos. Os ciclos hidrológicos são afetados pelas mudanças naturais e por aquelas produzidas pelo homem, como, por exemplo, o uso excessivo dos aquíferos para abastecimento ou irrigação (Vörösmarty et al., 2010; McNutt, 2013; Young et al., 2014).

\section{PRINCIPAIS PROBLEMAS E CONEXÕES DA CRISE HÍDRICA}

\section{Ameaças causadas por enchentes e extremos de precipitação}

Enchentes de diferentes frequências, intensidades e durações ocorrem em distintas regiões por causas diversas. Algumas enchentes podem ser antecipadas com predições sobre volume. Outras não podem ser preditas e causar inúmeros desastres e ameaças à segurança coletiva da população. Os custos dos danos produzidos por enchentes em áreas rurais e urbanas são muito elevados; com enchentes ocorrem, em muitas regiões, deslizamentos, danos à propriedade e à vida humana (UN-WWAP, 2006).

\section{Ameaças decorrentes da seca}

Muitas atividades humanas e danos aos ecossistemas aquáticos (águas superficiais, rios, lagos, represas, pântanos) ocorrem com secas prolongadas quando a precipitação é significantemente mais baixa que o normal para determinada região.

Nas regiões urbanas, essa seca atinge principalmente o abastecimento público, mas outros usos, como produção de energia, produção de alimentos e navegação, podem ficar comprometidos. Por exemplo, na seca de 2013-2014 no Sudeste do Brasil, que afetou aproximadamente 80 milhões de pessoas nos estados de Minas Gerais, São Paulo e Rio de Janeiro, além de problemas no abastecimento público, a hidrovia do Tietê ficou desativada por quatro meses, resultando em perda de $5 \mathrm{mil}$ postos de trabalho e milhões de toneladas de materiais não transportados.

Secas produzem impactos econômicos severos e de lenta recuperação, com efeitos na produção industrial e resultando em inúmeros problemas econômicos adicionais. A seca de 2007-2008 em Barcelona, na Espanha, produziu prejuízos econômicos de 1 bilhão de euros e reduziu em $1 \%$ o produto interno bruto da Catalunha, região economicamente avançada da Espanha.

\section{Ameaças resultantes da deterioração da qualidade da água}

Com a crise hídrica, resultante de enchentes ou secas, há uma deterioração da qualidade das águas superficiais e subterrâneas. Tundisi et al. (2015) demonstraram como, a partir da segunda metade do século XIX, ocorreu uma contínua e inexorável deterioração da qualidade das águas superficiais e subterrâneas aumentando a complexidade ana- 
lítica e a toxicidade. A diminuição do volume de reservatórios, lagos e rios, com o aumento das concentrações de fósforo, nitrogênio e de substâncias tóxicas, como pesticidas, herbicidas e compostos orgânicos, resulta em maior toxicidade, acentuando os riscos à saúde humana.

$\mathrm{O}$ aumento da temperatura do ar, como resultado das mudanças globais, pode elevar a temperatura da água a $1^{\circ} \mathrm{C}$ ou $2^{\circ} \mathrm{C}$ acima das médias históricas, o que estimula o crescimento de cianobactérias e vírus de grande impacto na qualidade das águas e na saúde humana. Essa sinergia foi mostrada por Tundisi et al. (2015) para ilustrar os efeitos da seca em um reservatório raso subtropical situado no centro do estado de São Paulo (Represa da UHE Carlos Botelho/Lobo-Broa).

\section{Água, saúde humana e crise hídrica}

As fontes de contaminação de águas são inúmeras, consistindo de resíduos domésticos orgânicos, poluição industrial e agrícola. Poluição térmica devido a efluentes industriais de refrigeração é outra fonte, estimulando o desenvolvimento de patógenos, bactérias e vírus. Todos esses componentes têm um efeito considerável na saúde humana. Além das doenças de veiculação hídrica resultantes do desenvolvimento de vetores, como as larvas de mosquitos transmissores de dengue e malária, ou gastroenterites causadas por vírus, protozoários e bactérias, o complexo conjunto de substâncias orgânicas dissolvidas, como pesticidas e herbicidas, cosméticos, remédios e hormônios, pode ter efeitos diretos e indiretos na saúde humana, ainda não totalmente identificados (Tundisi et al., 2015).

Com as crises hídricas, ocorrem alterações do volume e da concentração de nutrientes nas secas e, durante as enchentes, essas condições podem se agravar e introduzir novos problemas de controle, avaliação e detecção dos impactos na saúde humana.

Florações de cianobactérias em represas de abastecimento têm ocorrido com grande frequência em função do aumento da temperatura da superfície da água, diminuição do volume devido a menor precipitação e aumento relativo da concentração de nutrientes dissolvidos na água (Confalonieri, Heller \& Azevedo, 2010). Portanto, além de alterar a quantidade de água, as crises hídricas afetam a qualidade e, consequentemente, aumentam os impactos à saúde humana com efeitos econômicos diretos e indiretos, ainda pouco contabilizados.

\section{IMPACTOS ECONÔMICOS E SOCIAIS}

As águas doces são um recurso fundamental para a saúde humana e a prosperidade e segurança coletiva da população. Água de boa qualidade é essencial para a erradicação de pobreza, segurança alimentar e preservação dos ciclos biogeoquímicos e ecossistemas. A dependência da economia em relação à água é reconhecida em muitos estudos e avaliações (Eliasson, 2015; Young et al., 2015). A produção de alimentos pode ser afetada pela crise hídrica, diminuindo a oferta e reduzindo estoques. Em países onde a hidroeletricidade tem papel fundamental na produção de energia, como no Brasil, a crise hídrica pode atingir essa produção, reduzindo a oferta. Usos competitivos de água em reservatórios de produção de energia podem resultar em conflitos e perdas econômicas. A redução dos volumes dos reservatórios do sistema hidráulico do Rio Paraíba do Sul, descrita por Galvão e Berman (2015), resultou em redução da produção de energia e adequação e reformulação do abastecimento de água.

A redução geral do volume útil de reservatórios diminui a produção de energia hidrelétrica, aumenta o uso de energia termelétrica (com aumento das emissões de gases de efeito estufa) e compromete outros usos competitivos dos reservatórios, como o abastecimento público, a pesca, a agricultura e a recreação. Esta última atividade tem um papel econômico ainda não totalmente mensurado e reconhecido no Brasil. Águas interiores são intensamente utilizadas para a recreação, e o impacto da crise hídrica com a deterioração da qualidade das águas impede a recreação, com reflexos econômicos em muitos municípios próximos a represas e rios. Impactos sociais decorrentes desses processos ocorrem devido à perda de postos de trabalho, à disrupção de cadeias produtivas regionais e à instabilidade social causada por insegurança coletiva resultante da escassez, da falta de acessibilidade à água e vulnerabilidade devido à baixa qualidade da água (Tundisi, 2014, comunicação ao Ministério Público do Estado de São Paulo). 


\section{ÁGUA PARA 0 \\ DESENVOLVIMENTO SOCIAL}

Água é essencial para o desenvolvimento social, o que inclui educação, facilidade de acesso a saneamento básico e água de boa qualidade. Em muitos países em desenvolvimento, a existência de escolas sem instalações sanitárias adequadas é motivo de exclusão social e elemento importante de exclusão de gênero (Young et al., 2015).

\section{ÁGUA PARA ATENDER ÀS NECESSIDADES BÁSICAS DA POPULAÇÃO}

Água em quantidade adequada e de boa qualidade é de fundamental importância para o abastecimento, a saúde humana, a produção de alimentos (Goodfray et al., 2010) e a qualidade de vida em geral. A crise hídrica pode afetar esses aspectos básicos de sobrevivência humana.

\section{ÁGUA PARA 0 DESENVOLVIMENTO ECONÔMICO}

A água é essencial e um recurso estratégico para o desenvolvimento econômico, para a produção de energia e a produção industrial. Sendo um recurso natural essencial para a espécie humana e para o funcionamento dos ecossistemas, seu valor econômico e sua importância estratégica são reconhecidos como promotores das economias locais, regionais, das bacias hidrográficas e dos continentes. Usos da água para a produção de biocombustível têm aumentado consideravelmente em alguns países com impactos nas economias regionais. Há uma relação direta de causa e efeito entre água e desenvolvimento econômico (Jimenez-Cisneros, 2015).

\section{A ÁGUA E OS ECOSSISTEMAS NATURAIS}

A água é fundamental para a manutenção e a sustentabilidade dos ecossistemas naturais e dos ciclos biogeoquímicos e de biodiversidade, os quais são cruciais para a própria sobrevivência da espécie humana e de sua qualidade de vida (Unep, 2006).

A manutenção dos fluxos ecológicos nas crises hídricas deve reduzir a vulnerabilidade dos ecossistemas e impedir a disrupção dos ciclos, promovendo a preservação da qualidade e quantidade, da biodiversidade e dos serviços ecossistêmicos.

\section{AS SOLUÇÕES PARA ENFRENTAR AS CRISES HÍDRICAS}

A severidade e a complexidade das crises hídricas, as quais deverão ainda persistir por longos períodos, demandam soluções que vão desde planejamento estratégico de longo prazo (Cortes \& Torrente, 2015) até medidas estruturais e de governança avançada. As diferentes soluções aqui apresentadas têm como referência trabalhos recentes (IEA, 2015; Jimenez-Cisneros, 2015; Young et al., 2015) e documentos e avaliações sobre recursos hídricos e tecnologias de aplicação (McClain, Du Bowy \& Zalewski, 2013; Moss et al., 2013; Tundisi, 2014).

É importante destacar a complexidade e as inúmeras conexões ecológicas, econômicas e sociais de crise hídrica para propor soluções que englobem esses processos e tenham foco no futuro.

\section{Redução de demanda}

$\mathrm{O}$ aumento de demanda e a diversificação dos usos múltiplos intensificaram a crise hídrica. A redução da demanda doméstica e industrial é um imperativo e deve ser orientada por comunicação adequada, novos processos de gestão e mobilização e tecnologia avançada. Tecnologias para a redução da demanda doméstica devem ser incentivadas (Jimenez-Cisneros, 2015).

\section{Reúso de água}

O tratamento de esgotos induzirá ao reúso de água para diferentes finalidades e proporcionará economia de água e impactos nas economias regionais e até nacionais dependendo do volume de reúso. Para tanto é necessário estabelecer no Brasil padrões para água de reúso, ampliando sua 
capacidade e o tratamento de esgotos (Hespanhol, 2010; Tundisi, 2014).

\section{Redução das perdas de água na rede}

A média no Brasil é de $30 \%$ de perda na rede. A renovação da infraestrutura com tecnologias mais avançadas para a redução das perdas certamente contribuirá para o processo de gestão integrada e econômica. É necessário um estímulo para inovação no desenvolvimento de novas tecnologias para redução das perdas de água (Tundisi, 2014).

\section{Avanços na governança de água}

A adoção da bacia hidrográfica como unidade de gestão e a gestão integrada dos recursos hídricos com governança transparente, participativa e com amplas bases tecnológicas são fundamentais como recurso para enfrentar as crises hídricas (Likens, 1992).

É necessário que a gestão seja apoiada com uma ampla base de dados que inclua inclusive séries históricas climatológicas (Cortes \& Torrente, 2015). A gestão hídrica por bacias hidrográficas no estado de São Paulo é complexa e pouco efetiva (Jacobi, Cibim \& Leão, 2015), o que se repete também em outros estados do Brasil (Tundisi, 2014).

É provável que a implementação de agências de bacia possa acelerar o processo de consolidação da gestão por bacias hidrográficas (Nakamura \& Rast, 2011), sendo que a governança da água deve incluir a capacidade de promover políticas públicas e organização institucional aceitável pela sociedade com a mobilização de recursos sociais que possam suportar a implementação dessas políticas públicas.

De acordo com Rogers (2006), nas bacias hidrográficas a sociedade deve ter capacidade de governança necessária para promover os ajustes e avanços na gestão integrada. A capacidade de regulação na bacia hidrográfica deve ter condições para integrar quantidade e qualidade de água e tratar dos problemas de águas superficiais, águas subterrâneas com a mensuração de suprimento, demanda e monitoramento da qualidade. Para o autor, a governança de água, a partir das bacias hidrográficas, deve ter quatro atributos fundamentais: ser aberta e transparente; inclusiva e comunicativa; coerente e integradora; equilibrada e ética. A operação da governança de água deve ser eficiente, sustentável e confiável.

\section{Tecnologias}

Há um conjunto enorme de tecnologias avançadas que podem ser introduzidas rapidamente e que tornam a gestão antecipada das crises hídricas eficiente. Dentre essas tecnologias estão: novos métodos de monitoramento de qualidade da água em tempo real, associados ao uso de imagens de satélite (Ogashawara et al., 2014; Tundisi et al., 2015); reflorestamento maciço de mananciais com espécies nativas, utilizando-se o princípio da remuneração por serviços ambientais; uso do conceito de eco-hidrologia e integração de biogeosfera com as demandas da sociedade (Hupfer, Figueiredo \& Tundisi, 2013); restauração de ecossistemas urbanos como os rios (Anelli, 2015) e implantação de parques municipais como mecanismo de reposição de águas na atmosfera e recarga de aquíferos (Buckeridge, 2015; Tundisi, 2014); unificação dos dados em saúde humana, qualidade das águas, usos e ocupação do solo em áreas urbanas com a finalidade de promover gestão avançada de água em sistemas urbanos (ABC/ Die Junge Akademie/Leopoldina Nationale Akademie der Wissenchaften, 2014).

A combinação da engenharia com a ecologia para promover soluções integradas é uma das soluções tecnológicas avançadas (Jimenez-Cisneros, 2015).

\section{Preservação das águas subterrâneas}

Com o aumento das pressões sobre as águas superficiais, indústrias e sistemas urbanos de abastecimento voltam-se para o suprimento de águas subterrâneas, as quais já se encontram sob ameaça em muitas regiões (Llamas \& Martinez-Santos, 2005). A preservação do uso excessivo dos mananciais subterrâneos e a prioridade para proteção de sua qualidade são fundamentais para manter uma reserva crítica e sustentável de enorme importância para a gestão integrada de recursos hídricos (Hirata, Zoby \& Oliveira, 2010; McNutt, 2013). 


\section{Saneamento básico e gestão integrada de recursos hídricos}

A gestão de resíduos sólidos é também de extrema importância na gestão integrada de recursos hídricos. Saneamento básico universal e acessibilidade à água são fundamentais para a saúde humana especialmente nas pressões durante as crises hídricas (Jasny et al., 2014).

\section{Educação e capacitação de recursos humanos}

A educação em geral da população e as interações sobre dimensão, complexidade, persistência e efeitos das crises hídricas devem fazer parte da política hidrossocial de municípios e organizações estaduais e federais. Igualmente crítica é a capacitação de técnicos, gestores e pesquisadores com uma visão sistêmica dos recursos hídricos e suas conexões e articulações com os ciclos hidrogeoquímicos, hidroeconômicos e hidrossociais (Young et al., 2015).

\section{CONCLUSÕES}

Se considerarmos as inter-relações entre recursos naturais (água, solo, ecossistemas, biodiversidade, recursos marinhos, recursos minerais não renováveis), sistema climático (temperatura, precipitação, variações e extremos climáticos, cobertura de gelo, correntes oceânicas e nível de oceanos), segurança humana (água, alimento, energia, saúde, renda e qualidade de vida) e estabilidade social (eventos políticos, migração, violência, conflitos, cooperação, instituições) (Scheffran et al., 2012), pode-se aquilatar a intensidade, a complexidade e o enorme impacto das crises hídricas. As interdependências entre estabilidade social, vulnerabilidade ambiental e segurança humana sob os efeitos das funções de força, sensibilidade ao clima e ações humanas são claras. A emergência de conflitos humanos resultantes dessas inter-relações só tende a se agravar com a persistência e o aprofundamento da crise hídrica e ambiental.

A responsabilidade dos cientistas, das organizações sociais e das autoridades governamentais é evidente, sendo necessário um agrupamento e foco nos esforços e cuja mensagem aos governos é direta: eles devem utilizar muito mais as informações científicas e os dados que eles mesmos financiam e que, por negligência, visão política atrasada ou ausência de projeto nacional, estadual ou municipal, não incorporam no planejamento estratégico de longo prazo. Se é que isso existe. De todo modo, o planejamento estratégico para o futuro, assegurando qualidade de vida adequada à população, é papel fundamental e obrigação dos governos em todos os níveis (Tundisi et al., 2015). 


\section{BIBLIOGRAFIA}

ABC - Academia Brasileira de Ciências/ Die Junge Akademie/Leopoldina Nationale Akademie der Wissenchatten. Water in Urban Regions: Building Future Knowledge to Integrate Land Use, Ecosystem Services and Human Health. Berlin, 2014.

ANELLI, R. L. S. “Uma Nova Cidade para as Águas Urbanas", in Estudos Avançados, v. 29(84), 2015, pp. 69-84.

BUCKERIDGE, M. "Árvores Urbanas em São Paulo: Planejamento Economia e Água", in Estudos Avançados, v. 29(84), 2015, pp. 85-101.

CONFALONIERI, U.; HELLER, L.; AZEVEDO, S. "Água e Saúde: Aspectos Globais e Nacionais", in C. Bicudo; J. G. Tundisi; M. Scheuenstuhl (orgs.). Águas do Brasil: Análises Estratégicas. Academia Brasileira de Ciências, Instituto de Botânica, 2010, pp. 27-38.

CORTES, L. P.; TORRENTE, M. “Crise de Abastecimento de Água em São Paulo e Falta de Planejamento Estratégico", in Estudos Avançados, v. 29(84), 2015, pp. 7-26.

ELIASSON, J. "The Rising Pressure of Global Water Shortages", in Nature 517(7532), 2015. Disponível em: http://www.nature.com/news/the-rising-pressure-of-global-watershortages-1.16622.

GOODFRAY, C. J. et al. "Food Security: the Challenge of Feeding 9 Billion People", in Science, v. 327, 2010, pp. 812-7.

HESPANHOL, I. "Conservação e Reúso como Instrumentos de Gestão para Atenuar os Custos de Cobrança pelo Uso de Água no Setor Industrial", in C. Bicudo; J. G. Tundisi; M. Scheuenstuhl. Águas do Brasil. Análises Estratégicas. Academia Brasileira de Ciências, Instituto de Botânica, 2010, pp. 59-80.

HIRATA, H.; ZOBY, J. L. G.; OLIVEIRA, F. R. "Água Subterrânea: Reserva Estratégica ou Emergencial", in C. E. M. Bicudo; J. G. Tundisi; M. Scheuenstuhl (orgs.). Águas do Brasil: Análises Estratégicas. Academia Brasileira de Ciências, Instituto de Botânica, 2010, pp. 149-61.

HUPFER, H. M.; FIQUEIREDO, J. A. S.; TUNDISI J. G. (orgs.). Pagamento por Serviços Ambientais. Porto Alegre, Entremeios, 2013.

JASNY, B. et al. "Global Health. What Works", in Science, v. 345, issue 6.202, 2014, p. 1.256. JACOBI, P. R.; CIBIM, J.; LEÃO, R. S. “Crise Hídrica na Macrometrópole Paulista e Respostas da Sociedade Civil", in Estudos Avançados, v. 29(84), 2015.

JIMENEZ-CISNEROS, B. "Responding to the Challenges of Water Security: the Eighth Phase of the International Hydrological Programme 2014-2021", in C. Cudennec et al. Hydrological Sciences and Water Security: Past, Present and Future. PIAHS 366, 2015, pp. 10-9.

LLAMAS, R.; MARTINEZ-SANTOS, P. "Intensive Groundwater Use: Silent Revolution and Potential Source of Social Conflict", in ASCE Journal of Water Resources Planning and Management, 131(5), 2005, pp. 337-41.

MCCLAIN, M. E; DU BOWY, P. J.; ZALEWSKI, M. “Ecohydrology for the Harmonization of Societal Needs with the Biosphere Potential", in Ecohydrology \& Hydrobiology, v. 13, 2013, pp. 3-5.

McNUTT, M. "The Drought You Can't See", in Science, v. 345, issue 6.204, 2013, p. 1.543.

MOSS, B. et al. "Synthesis and Conclusions to the International Symposium on Ecohydrology, Biotechnology and Engineering: Torwards Harmony between the Biogeosphere and Society on the Basis of Long-Term Ecosystem Research", in Ecohydrology \& Hidrobiology, v. 14(1), 2014, pp. 2-13. 
NAKAMURA, M.; RAST, W. "Development of Integrated lake Basin Management Process", in Research Caster for Sustainability and Environment. Japan, Shiga University, 2011.

OGASHAWARA, I. et al. "Cyanobacteria Detection in Guarapiranga Reservoir (S. PauloBrazil) Using Landsat T. M. and ETM Images", in Ambiente \& Água, v. 9, n. 2. Taubaté, pp. 1-14.

ROGERS, P. P. "Water Governance, Water Security and Water Sustainability”, in P. P. Rogers; N. Ranon Llanas; L. Martinez-Cortina (eds.). Water Crisis: Myth or Realty?. London, Fundación Marcelino Botín/Taylor Francis, 2006.

SCHEFFRAN, J. et al. "Climate Change and Violent Conflict", in Science, v. 336, 2012, pp. 86971.

TUNDISI, J. G. (coord.). Recursos Hídricos no Brasil: Problemas, Desafios e Estratégias para o Futuro. Estudos Estratégicos. Rio de Janeiro, Academia Brasileira de Ciências, 2014. "Cianobactérias e Qualidade da Água na Represa da UHE Carlos Botelho (Lobo/ Broa)", in Relatório ao Ministério Público do Estado de São Paulo, 2014. "Entrevista", in Science, v. 347, issue 6.224, 2015, pp. 812.

TUNDISI, J. G. et al. "Water Availability, Water Quality, Water Governance: the Way Ahead", in C. Ardenec et al. (ed.). Hydrological Sciences and Water Security: Past, Present and Future. PIAHS, 2015, pp. 75-9.

TUNDISI, J. G. et al. "A Bloom of Cyanobacteria (Cylindrospermopsis raciborskii) in UHE Carlos Botelho (Lobo/Broa) Reservoir: a Consequence of Global Change?", in Brazilian Journal of Biology, v. 75, n. 2, 2015, pp. 507-8.

UNEP - United Nations Environment Programme. Marine and Coastal Ecosystems and Human Well-being: a Synthesis Report Based on the Findings of the Millennium Ecosystem Assessment. Nairobi, Unep, 2006. Disponível em: http://www.unep.org/pdf/ Completev6_LR.pdf.

UN-WWAP (United Nations Water Assessment Programme) UN World Water Development Report 2: Water, a Shared Responsibility. Paris, New York and Oxford. Unesco (United Nations Educational, Scientific and Cultural Organization) and Berghahn Books. 2006. Disponível em: http://unesdoc.unesco.org/images/0014/001444/144409E.pdf.

UNESCO - United Nations Educational, Scientific and Cultural Organization. International Hydrological Programme - IHP. $20^{\text {th }}$ Session of the Intergovernmental Council. Paris, June 2012. Disponível em: http://unesdoc.unesco.org/images/0021/002164/216434E.pdf.

VÖRÖSMARTY, C. J. et al. "Global Threats to Human Water Security and River Biodiversity", in Nature, 467(7.315), 2010, pp. 555-61.

WATSON, R. T.; ZINYOWERA, M. C.; MOSS, R. H. (eds.). The Regional Impacts of Climate Change: an Assessment of Vulnerability. Intergovernmental Panel on Climate Change IPCC. Cambridge University Press, 1998.

YOUNG, G. et al. "Hydrological Challenges and Water Security: an Overview", in Hydrological Sciences and Water Security: Past, Present and Future. Proceedings of the 22th FOUACS Collegium. Paris, June, 2014, IHHS Publ., 2015, pp. 2-9. 